\title{
Celiac Disease in 2015: Where are we now? Where do we go from here?
}

\author{
Furqaan Ahmed, MD* \\ South City Hospital, Karachi, Pakistan
}

Received: August 11, 2015; Accepted: September 10, 2015; Published: September 21, 2015

*Corresponding author: Furqaan Ahmed, South City Hospital, 128C KDA Scheme 1, Karachi, Pakistan. Tel- 92-300-825-9667, E-mail: furqaan@hotmail.com

\begin{abstract}
Celiac disease is an immune-mediated systemic disease precipitated by the ingestion of gluten-containing foods in genetically susceptible persons. Gastrointestinal symptoms are common. The diagnosis of celiac disease is based on the presence of typical autoantibodies, characteristic histologic changes on small intestinal biopsy, and clinical improvement in response to a gluten-free diet. The natural history of the celiac disease varies widely. The management of celiac disease includes the institution of a glutenfree diet. Intestinal damage heals within 6 to 24 months after institution of a gluten-free diet. Celiac disease exists on a spectrum of gluten-related disorders. Several important research studies were published in 2014. An increasing rate of non-classical and subclinical phenotypes compared to the classical one was described. Larazotide acetate, an oral peptide which closes tight junction's thus reducing gluten uptake, shows promise as an adjuvant therapy for patients who continue to have symptoms despite efforts to avoid gluten. There are multiple unresolved questions surrounding celiac disease and the spectrum of gluten-related disorders.
\end{abstract}

\section{Introduction}

Celiac disease is an immune-mediated systemic disease precipitated by the ingestion of gluten-containing foods in genetically susceptible persons. Celiac disease affects 0.6 to $1.0 \%$ of the population with wide regional differences $[1$, 2]. The prevalence of celiac disease is rising due, in part, to the westernization of diet in developing countries and due to increased awareness. It occurs due to activation of both cellmediated ( $\mathrm{T}$ cell) and humoral (B cell) immune responses in response to gluten ingestion. Gluten is found in wheat, barley, and rye.

Genetic predisposition is seen, with most patients expressing the HLA-DQ2 haplotype. First and second-degree relatives have an increased risk of celiac disease. However, genetic susceptibility alone is not enough for the loss of gluten tolerance. Celiac disease epidemiology has iceberg characteristics with far more undiagnosed cases than diagnosed ones.

In adults, celiac disease is diagnosed an average of ten years after the onset of symptoms. Gastrointestinal symptoms, while common, are not mandatory, and include chronic diarrhea, abdominal distension, iron deficiency anemia, and weight loss. Non gastrointestinal symptoms include peripheral neuropathy, fatigue, reduced bone mineral density, dermatitis herpetiformis, aphthous stomatitis, high aminotransferase levels, folate deficiency, and infertility. Celiac disease can be classified as classical (predominately gastrointestinal symptoms), atypical (predominately non gastrointestinal symptoms), and silent (asymptomatic despite the presence of characteristic intestinal lesions).

The initial screening for celiac disease should be the measurement of IgA-tissue transglutaminase antibodies due to its high sensitivity and specificity, although it performs less well in patients with IgA deficiency. The diagnosis of celiac disease is based on the presence of typical autoantibodies, characteristic histologic changes on small intestinal biopsy, and clinical improvement in response to a gluten-free diet. Characteristic histologic findings graded using the Marsh classification, include villous atrophy, an increased number of intraepithelial lymphocytes, and crypt elongation.

The natural history of the celiac disease varies widely. To a variable extent, the following sequence of events may occur- the presence of serologic markers, the development of intestinal enteropathy, symptom onset, and the development of complications. Patients with active celiac disease have an increased risk of death compared to the general population; a risk that returns to normal after 3-5 years of a strict glutenfree diet. Complications of longstanding, untreated celiac disease include malignant, small bowel neoplasia, large intestine adenocarcinoma, osteoporosis, stunted growth, and infertility.

The management of celiac disease includes the institution of a gluten-free diet, referral to a dietician and support group (the lifelong elimination of dietary gluten has social and psychological consequences), screening for iron and folate deficiency, consideration of bone density testing, and counseling regarding the serological screening of first- and second-degree relatives. Intestinal damage heals within 6 to 24 months after institution of a gluten-free diet. Celiac disease patients require monitoring to follow adherence to dietary restrictions and for the development of associated conditions and complications. 


\section{Guidelines}

In recent years, multiple societies have published guidelines regarding the diagnosis and management of celiac disease. No new major guidelines were published in 2014.

The American College of Gastroenterology published celiac disease guidelines in 2013 [3]. Salient recommendations from these guidelines include the following:

1. IgA tissue transglutaminase testing is preferred, except in IgA deficiency.

2. HLA-DQ2 and HLA-DQ8 genetic testing is useful in select circumstances, particularly in patients with a discrepancy between histology and serology, and those with suspected refractory celiac disease.

3. Test results may be misleading if a patient is already on a gluten-free diet.

4. Celiac disease has a high prevalence (10\%) in Down syndrome.

5. Biopsies are critical; 1-4 from the duodenal bulb and four postbulbar biopsies should be obtained.

6. Other causes of lymphocytic infiltration should be considered.

7. Celiac disease should also be considered in patients with abnormal liver enzymes.

8. Involvement of a dietician is essential.

The European Society for Pediatric Gastroenterology, Hepatology, and Nutrition (ESPGHAN), the World Gastroenterology Organizations, American Gastroenterology Association, and National Institute of Health have also published similar guidelines [4-7].

\section{Spectrum of Gluten-Related Disorders}

Celiac disease exists on a spectrum of gluten-related disorders. Recently, a panel of experts published a consensus on new nomenclature and classification of gluten-related disorders [8]. There are three primary categories of gluten-related disease. Celiac disease occurs due to autoimmune mechanisms. A second mechanism of gluten-related disease is allergy-mediated. In wheat allergy, cross-linking of IgA by repeat sequences in gluten peptides leads to histamine release from basophils and mast cells. Non-allergic and non-immune mediated gluten disorders are called gluten sensitivity. Gluten sensitivity likely includes a variety of different conditions, as yet to be clearly identified, but characterized by an adverse reaction to dietary gluten. The consensus panel definition of non-celiac gluten sensitivity is "a term that relates to one or more of a variety of immunological, morphological, or symptomatic manifestations that are precipitated by gluten in individuals in whom celiac disease has been excluded." These conditions may include intolerance to the carbohydrate component of gluten (fructose malabsorption) or protein intolerances (amylase trypsin inhibitors). Gluten sensitivity is further characterized by a shorter duration between gluten exposure and symptoms, no HLA-DQ2 or HLA DQ8 restrictions, no autoantibodies or enteropathy, and an absence of long-term complications.

\section{New Data Published in 2014}

Several interesting and important research studies were published in 2014. Genetic predisposition, particularly an association of the HLA haplotype DR3-DQ2 and DR4-DQ8, has been described as one risk factor contributing to the development of celiac disease. Edwin Liu and colleagues published a study in the New England Journal of Medicine following 6403 children with these HLA haplotypes prospectively from birth and found them to be at high risk for celiac disease in childhood [9]. Geographic variation was noted, suggesting the importance of environmental factors. Another study found a higher prevalence of celiac disease in patients with autoimmune hepatitis, using the largest cohort studied to date [10].

A study of 770 celiac disease patients over a 15 year period at an Italian referral center found a changing clinical profile of celiac disease over time [11]. Over the study period, an increasing rate of non-classical (Extraintestinal and/or gastrointestinal symptoms other than diarrhea) and subclinical phenotypes compared to the classical (malabsorption syndrome) one. Separately, Biesiekierski and colleagues reported that most adults with selfdiagnosed non-celiac gluten sensitivity do not meet the criteria for this diagnosis [12].

Several studies published in 2014 looked at differentaspects of the pathophysiology of the celiac disease. Peterson and colleagues found an association between T-cell receptor recognition of HLADQ2 gliadin complexes and celiac disease [13]. Another study reported specific non gluten proteins of wheat (serpins, purinins, $\alpha$-amylase/protease inhibitors, globulins, and farinins) as non gluten targets of the humoral response seen in celiac disease [14]. It has been previously suggested that antibiotic exposure in pregnancy may increase the risk of celiac disease in offspring. A study by Marild et al found no such association [15].

Another study described the role of intestinal microbiota in gluten metabolism, offering a possible new therapeutic avenue for further study [16]. Kurppa and colleagues found a significant benefit of a gluten-free diet in asymptomatic patients with serologic markers for celiac disease [17].

Perhaps the most significant development in the therapy of celiac disease in 2014 was the study presented by Joseph Murray and colleagues as a late-breaking abstract at Digestive Disease Week 2014 [18]. This study reported the results of a randomized, parallel, double-blind, placebo-controlled, multicenter trial of larazotide acetate. This oral peptide closes the tight junctions which are compromised in celiac disease, thus reducing gluten uptake. The study reported a significant reduction of symptoms. This new drug is meant to supplement, not replace, the glutenfree diet which remains the cornerstone of celiac disease therapy. It may be particularly useful in patients who continue to have symptoms despite efforts to avoid gluten. 


\section{Controversies and Areas for further Research}

There are multiple areas of controversy surrounding celiac disease and the spectrum of gluten-related disorders. Celiac disease occurs in response to gluten exposure in genetically susceptible persons, after as yet undetermined environmental triggers. Proposed environmental factors include the age of dietary gluten introduction, the composition of gut flora, breast feeding, and intestinal infections.

Other areas that require further study include:

- How best to assess compliance with a gluten-free diet.

- Whether oats need to be excluded from a gluten-free diet.

- The benefits and cost-effectiveness of population-based screening for celiac disease.

- The prevalence, pathophysiology, and natural history of non-celiac gluten sensitivity.

- Further discrimination between celiac disease, non-celiac gluten sensitivity, and what allergy.

- Identification and discrimination between the likely various conditions presently grouped under the umbrella of nonceliac gluten sensitivity.

- Updating the original classification of celiac disease mucosal pathology published by Marsh [19, 20].

- Better understanding, using large population-based prospective studies, of the link between celiac disease and various autoimmune disorders, and the effects of a glutenfree diet on these associated autoimmune conditions.

- Larger scale studies with larazotide acetate and other therapeutic agents.

\section{Summary}

Of the spectrum of gluten-related disorders celiac disease, an immune-mediated systemic disease, is the best characterized and understood. Gluten avoidance remains the cornerstone of therapy but new compounds are under investigation for adjuvant therapy. Many unresolved questions remains. Furthermore, other disease states in the spectrum of gluten-related disorders need to be more explicitly elucidated.

\section{References}

1. Fasano A, Berti I, Gerarduzzi T, Not T, Colletti RB, Drago S, et al Prevalence of celiac disease in at-risk and not-at-risk groups in the United States: a large multicenter study. Arch Intern Med. 2003;163(3):286-292.

2. Biagi F, Klersy C, Balducci D, Corazza GR. Are we not over-estimating the prevalence of celiac disease in the general population? Ann Med. 2010;42(8):557-561. doi: 10.3109/07853890.2010.523229.

3. Rubio-Tapia A, Hill ID, Kelly CP, Calderwood AH, Murray JA. ACG Clinical guidelines: diagnosis and management of celiac disease. AM J Gastroenterol. 2013;108(5):656-676. doi: 10.1038/ajg.2013.79.
4. Hushy S, Koletsko S, Korponay-Szabo IR, Mearin ML, Phillips A, Shamir R et al. European Society for Pediatric Gastroenterology, Hepatology, and Nutrition guidelines for the diagnosis of celiac disease. J Pediatr Gastroenterol Nutr. 2012;54(1):136-160. doi: 10.1097/MPG.0b013e31821a23d0.

5. Bai J, Zeballos E, Fried M, et al. World Gastroenterology Organization practice guidelines: celiac disease. WGO 2007.

6. American Gastroenterological Association medical position statement: celiac sprue. Gastroenterology. 2001;120(6):1522-5.

7. NIH Consensus Development Conference on celiac disease. NIH Consens State Sci Statements 2004;21:1-23.

8. Sapone A, Bai JC, Ciacci C, Dolinsek J, Green PH, Hadjivassiliou M, et al. Spectrum of gluten-related disorders: consensus on new nomenclature and classification. BMC Medicine. 2012:10:13. doi: 10.1186/1741-7015-10-13.

9. Liu E, lee HS, Aronsson CA, et al. Risk of pediatric celiac disease according to HLA haplotype and country. N Engl J Med 2014:371(1):4249.

10. van Gerven NM, Bakker SF, de Boer YS, de Boer YS, Witte BI, Bontkes $\mathrm{H}$, et al. Seroprevalence of celiac disease in patients with autoimmune hepatitis. Eur J Gastroenterol Hepatol. 2014:26(10):1104-1107. doi: 10.1097/MEG.0000000000000172.

11.Volta U, Caio G, Stanghellini V, De Giorgio R. The changing clinical profile of celiac disease: a 15-year experience (1998-2012) in an Italian referral center. BMC Gastroenterology. 2014;14:194. doi:10.1186/ s12876-014-0194-x.

12. Biesiekierski JR, Newnham EC, Shepherd SJ, Muir JG, Gibson PR. Characterization of adults with a self-diagnosis of nonceliacgluten sensitivity. Nutr Clin Pract. 2014;29(4):504-509.

13. Peteron J, Montserrat V, Mujico JR, Loh KL, Beringer DX, van Lummel $\mathrm{M}$,et al. T-cell receptor recognition of HLA-DQ2-gliadin complexes associated with celiac disease. Nature Structural \& Molecular Biology. 2014;21(5):480-488. doi: 10.1038/nsmb.2817.

14. Huebener S, Tanaka CK, Uhde M, et al. Specific nongluten proteins of wheat are novel target antigens in celiac disease humoral response. J Proteome Res. 2014.

15. Marild k, Ludvigsson J, Sanz Y, Ludvigsson JF. Antibiotic exposure in pregnancy and risk of celiac disease in offspring: a cohort study. BMC Gastroenterol. 2014;14:75. doi: 10.1186/1471-230X-14-75.

16. Caminero A, Herran AR, Nistal E, Pérez-Andrés J, Vaquero L, Vivas S, et al. Diversity of the cultivable human gut microbiome involved in gluten metabolism: isolation of microorganisms with potential interest for celiac disease. FEMS Microbiology Ecology. 2014;88(2):309-319. doi: 10.1111/1574-6941.12295.

17. Kurppa K, Paavola A, Collin P, Sievänen H, Laurila K, Huhtala H, et al. Benefits of a gluten-free diet for asymptomatic patients with serologic markers for celiac disease. Gastroenterology. 2014;147(3):610617. doi: 10.1053/j.gastro.2014.05.003.

18. Wang C, Rasmussen H, Perrow W, et al. Larazotide Acetate, a First InClass, Novel Tight Junction Regulator, Meets Primary Endpoint and Significantly Reduces Signs and Symptoms of Celiac Disease in Patients on a Gluten-Free Diet: Results of a Multicenter, Randomized, Placebo Controlled Trial. Digestive Disease Week (DDW) 2014. Abstract 929b. Presented May 7, 2014 
19. Marsh MN. Gluten, histocompatibility complex and the small intestine. A molecular and immunobiologic approach to the spectrum of gluten sensitivity ('celiac sprue'). Gastroenterology. 1992;102(1):330-354.
20. Marsh MN, Cummins AG. The interactive role of mucosal T lymphocytes in intestinal growth, development and enteropathy.J Gastroenterol Hepatol. 1993;8(3):270-278. 